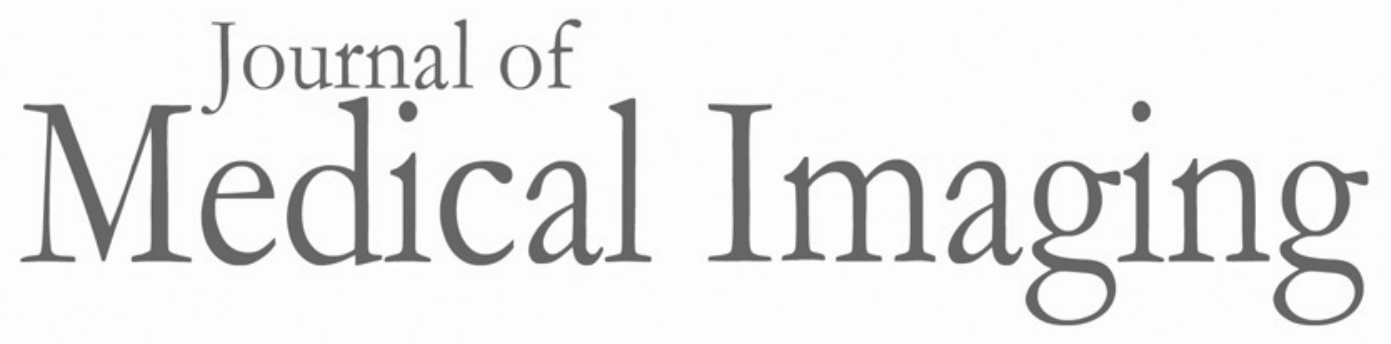

Medicallmaging.SPIEDigitalLibrary.org

\title{
Introduction to the LUNGx Challenge
}

\author{
Maryellen L. Giger
}

\section{SPIE.}




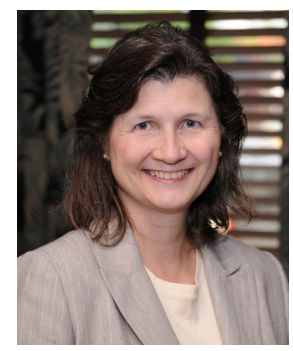

\section{Introduction to the LUNGx Challenge}

In this issue of the Journal of Medical Imaging (JMI), I invite you to read a peer-reviewed guest editorial from Sam Armato and team on "Reflections and Lessons Learned" from the SPIEAAPM-NCI Lung Nodule Classification Challenge, i.e., the LUNGx Challenge. The Challenge was a collaborative effort sponsored and supported by SPIE, the American Association of Physicists in Medicine (AAPM), and the National Cancer Institute $(\mathrm{NCl})$, and researchers from The University of Chicago, the University of Michigan, and Oak Ridge National Laboratory. The results from the Challenge were presented and discussed by a panel of experts at the SPIE Medical Imaging Symposium held February 22-26, 2015 in Orlando, Florida.

Medical imaging challenges can benefit the research community by allowing for direct comparisons of computer algorithms such that all algorithms abide by the same rules, are run on the same images, and are evaluated in the same manner and with the same metric. The task of the LUNGx Challenge was the computerized classification of lung nodules as benign or malignant on diagnostic computed tomography (CT) scans.

LUNGx was a successful scientific challenge, but it still had its own logistical challenges. The situation yielded valuable lessons for the organization of and participation in future challenges. In the guest editorial, Dr. Armato and team address aspects of establishing a challenge (such as providing an infrastructure for case dissemination, communication, and upload of consistently formatted results), data set considerations, clarity of challenge participation rules (including potential authorship), and participant responsibility. They note that perhaps the most important lesson is that organizers and participants have a mutual responsibility to approach a challenge with commitment and scientific rigor.

Maryellen L. Giger Editor-in-Chief 